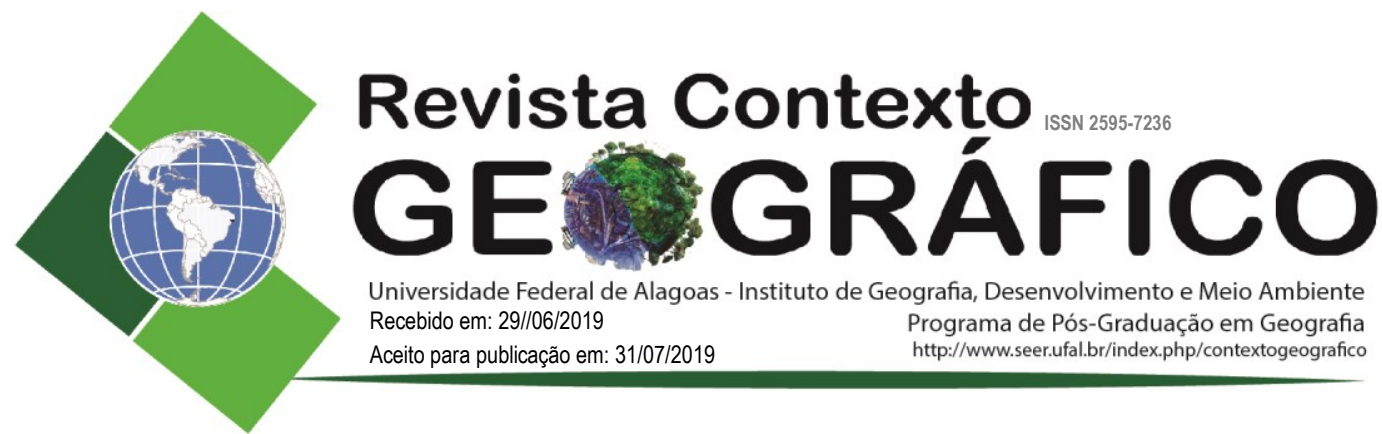

\title{
DIALOGOS Y VÍNCULOS EN LA CONSTRUCCIÓN DE UNA NUEVA GEOGRAFÍA EN EL CONO SUR EN LA DÉCADA DE 1970
}

\author{
Gonzalo Ezequiel Lus Bietti \\ Instituto de Geografía Romualdo Ardissone. Facultad de Filosofía y Letras. Universidad de \\ Buenos Aires \\ g.lus@hotmail.com
}

\begin{abstract}
RESUMEN - Este artículo pretende contribuir con la historia social de la Geografía, al analizar el desarrollo de un proyecto disciplinar de principios de la década 1970 que, inserto en un clima de agitación social, política y cultural, tanto en Argentina, Brasil y Uruguay, se construyó en contraposición a la Geografía regional hegemónica, buscando atender las problemáticas socioespaciales provenientes del desarrollo-subdesarrollo. En este sentido, además de conocer esta propuesta de Geografía en el Cono Sur, este trabajo indaga cuáles eran las bases teóricas que orientaban sus perspectivas de análisis y quiénes fueron los principales actores intervinientes, poniendo especial énfasis en saber qué vínculos mantuvieron entre sí. Para llevar adelante estos objetivos, se utilizó la técnica del análisis de contenido, interpretando fuentes teóricas, documentos y declaraciones. También se ha realizado una entrevista a uno de los actores claves. De acuerdo al análisis efectuado, en el Cono Sur tuvo lugar una Nueva Geografía comprometida con la realidad social, nacional y latinoamericana, cuyo sustento teórico encontró raíces en los trabajos de Pierre George, Yves Lacoste y/o Jean Tricart. Estas propuestas se cristalizaron en los Encuentros Latinoamericanos de la Nueva Geografía que estrecharon los vínculos y favorecieron la circulación de personas, ideas y materiales. Finalmente se propone comprender esta Nueva Geografía como un espacio de construcción de conocimiento transnacional que, a la luz del presente, también puede ser concebida como una primera expresión de la Geografía crítica en el Cono Sur.
\end{abstract}

Palabras-chave: Geografía Crítica-Década de 1970-Vínculos- Encuentros-Cono Sur.

\section{DIÁLOGOS E VÍNCULOS NA CONSTRUÇÃO DE UMA NOVA GEOGRAFIA NO CONE SUL NA DÉCADA DE 1970}

\begin{abstract}
RESUMO - Este artigo visa contribuir para a história social da Geografia, analisando o desenvolvimento de um projeto disciplinar do início dos anos 1970 que, inserido em um clima de agitação social, política e cultural, tanto na Argentina, quanto no Brasil e no Uruguai, foi construído, em contraste com a geografia regional hegemônica, buscando abordar os problemas sócio-espaciais decorrentes do desenvolvimento-subdesenvolvimento. Nesse sentido, além de conhecer essa proposta de Geografia no Cone Sul, este trabalho investiga quais foram as bases teóricas que nortearam suas perspectivas de análise e quais foram os principais atores envolvidos, com ênfase especial em saber quais os laços existentes entre eles. Para realizar esses objetivos, utilizou-se a técnica de análise de conteúdo, interpretando fontes teóricas, documentos e enunciados. Uma entrevista com um dos principais atores também foi realizada. De acordo com a análise realizada, no Cone Sul ocorreu uma Nova Geografia comprometida com a realidade social, nacional e latino-americana, cujo suporte teórico encontrou raízes nas obras de Pierre George, Yves Lacoste e / ou Jean Tricart. Essas propostas foram cristalizadas nos Encontros Latino-Americanos da Nova Geografia, que fortaleceram os vínculos e favoreceram o movimento de pessoas, ideias e materiais. Por fim, propõe-se entender essa Nova Geografia como espaço de construção do conhecimento transnacional que à luz do presente, também pode ser concebido como uma primeira expressão da Geografia crítica no Cone Sul.
\end{abstract}

Palavras Chaves: Geografia Crítica-Década de 1970-Vínculos-Encontros-Cone Sul.

\section{DIALOGUES AND LINKS IN THE CONSTRUCTION OF A NEW GEOGRAPHY IN THE SOUTHERN CONE IN THE 1970}




\begin{abstract}
This article aims to contribute to the social history of Geography, by analyzing the development of a disciplinary project of the early 1970s that, inserted in a climate of social, political and cultural agitation, both in Argentina, Brazil and Uruguay, were built in contrast to the hegemonic regional geography, seeking to address the socio-spatial problems arising from development-underdevelopment. In this sense, besides knowing this proposal of Geography in the Southern Cone, this work explores which were the theoretical bases that guided their perspectives of analysis and who were the main actors involved, with particular emphasis on knowing what links they had with each other. To carry out these objectives, the content analysis technique was used, interpreting theoretical sources, documents and statements. An interview with one of the key actors has also been conducted. According to the analysis made, in the Southern Cone took place a New Geography committed to the social, national and Latin American reality, whose theoretical support found roots in the works of Pierre George, Yves Lacoste and/or Jean Tricart. These proposals were crystallized in the Latin American Encounters of the New Geography that strengthened the links and favored the circulation of people, ideas and materials. Finally, it is proposed to understand this New Geography as a space for building transnational knowledge that, in the light of the present, can also be conceived as a first expression of critical Geography in the Southern Cone
\end{abstract}

Key Words: Critical Geography -1970s-Links-Encounters-Suouther Cone.

\title{
INTRODUCCIÓN
}

El siguiente artículo pretende contribuir con la historia social de la Geografía, al analizar el desarrollo de un proyecto disciplinar de fines de la década de 1960 y principios de 1970 que, inserto en un clima de agitación social, política y cultural, tanto en Argentina, Brasil y Uruguay, se construyó en contraposición a la Geografía regional hegemónica, buscando atender las problemáticas socio-espaciales provenientes del desarrollo-subdesarrollo y, en rigor, de la forma de organización del mundo capitalista. En efecto, consideramos que, a principios de la década del setenta, se configuró una Geografía en sintonía con las problemáticas de la época, y que, desde el cuestionamiento al paradigma hegemónico, planteó un proyecto disciplinar diferente, cuyas banderas serían el compromiso político con la realidad social, la integración latinoamericana, y la utilidad del conocimiento geográfico para la transformación de la sociedad. En este marco, nuestra labor no sólo pretende conocer esta propuesta de Geografía, sino que también buscará indagar cuáles son las bases teóricas que orientaban sus perspectivas de análisis y quiénes son los principales actores intervinientes, poniendo especial énfasis en saber qué vínculos tejieron y mantuvieron entre sí.

Para llevar adelante estos objetivos, nos posicionaremos desde la historia social de la disciplina, esto es, contextualizar histórica, social, política y culturalmente los episodios, autores e ideas geográficas (Livingstone, 1992). Como estrategia teórico-metodológica utilizaremos la técnica de análisis de contenido (Andréu Abela, 2003; Bernete García, 2013) analizando e interpretando tanto fuentes teóricas que orientan los trabajos de esta tendencia, como así también documentos, declaraciones y entrevistas realizadas a los actores claves. También, es de destacar que se realizó una entrevista semi-estructurada a Carlos Reboratti, importante geógrafo argentino del período.

Finalmente, como estructura de lectura, proponemos un primer apartado destinado a abordar algunos procesos históricos de la época que enmarcarían el desarrollo de la Geografía de Argentina, Brasil y Uruguay. En el segundo apartado, procuraremos analizar las propuestas geográficas que, desde la crítica a la Geografía regional hegemónica, emergerían en estos países y se cristalizarían en el proyecto de la Nueva Geografía. Así, por último, buscaremos conocer los principales encuentros y vínculos entre geógrafos y geógrafas que favorecerían la circulación de ideas y materiales para esta Nueva Geografía. A través de estos tres apartados intentaremos ver de qué manera se fue forjando un proyecto que, interactuando con su contexto y cuestionando a la matriz regional clásica, se convirtió en un espacio de producción de conocimiento transnacional, como así también en lo que, a la luz del presente, podríamos llamar una primera expresión crítica de la Geografía en el Cono Sur. 


\section{PENSAR EN/CON EL CONTEXTO}

Situarnos epocalmente en los años sesenta-setenta, nos obliga a tomar en consideración sucesos de variada índole que han contribuido a generar un clima de época. Focalizando en nuestro trabajo y acotando al espacio disponible, mencionaremos algunos procesos históricos cuyas implicancias políticas, sociales, culturales e intelectuales en Argentina, Brasil y Uruguay, podrían enmarcar el desarrollo de una primera Geografía crítica en el Cono Sur.

Durante los inicios de la década del sesenta, varios países de América Latina llevaron adelante un proyecto modernizador capitalista bajo la estrategia de la planificación y el desarrollo, a través del cual se buscaría consolidar una matriz productiva de orden industrial y nacional. Si bien este proceso tuvo particularidades en cada país del Cono Sur, en términos generales, no tardó en encontrar limitaciones. En efecto, hacia la segunda mitad de los sesenta, el desarrollismo encabezaría un debilitamiento en el que, o bien continuaría en forma conservadora, como sucedería en Argentina y Brasil, o bien terminaría por diluirse como en el caso de Uruguay (Nercesian, 2013). Las ideas en torno al desarrollismo habían encontrado su espacio de reflexión en la Comisión Económica para América Latina (CEPAL) desde 1947. En efecto, era por iniciativa de esta institución que se discutían y debatían temas vinculados tanto con las realidades nacionales como latinoamericanas, haciendo foco en las tensiones entre la industrialización y la producción agraria, el papel del Estado y las inversiones privadas en el desarrollo económico, como así también en la relación de las economías latinoamericanas con el mercado mundial. Con la consolidación de la Revolución Cubana, algunas de las ideas de la CEPAL, tomarían mayor carácter. En efecto, la experiencia de Cuba, a la vez que soslayaba la necesidad de una revolución que sacara a los países subdesarrollados de la dependencia de los desarrollados, presentaba a la misma idea de revolución como una posibilidad concreta y accesible (Nercesian, 2013). De esta manera, si los primeros años de la década del sesenta estaban atravesados por una teoría sobre la modernización capitalista; tras la Revolución Cubana, emergían otras teorías, como de la dependencia, el subdesarrollo y del Centro-Periferia, que ponían sobre la mesa que el subdesarrollo de unos países era causa y consecuencia del desarrollo de otros; y que el intento de expandir las economías latinoamericanas en base a capitales extranjeros sólo perpetuaba su condición de economía dependiente. En rigor, siguiendo a Oscar Terán (2008) tales teorías aparecían como resultado de una hibridación entre el cepalismo, el nacionalismo económico, el antiimperialismo y el marxismo.

Tras el triunfo de la experiencia cubana, y con la idea de revolución como una posibilidad concreta, desde Estados Unidos comenzó a haber cierto interés y preocupación por América Latina. En el marco de la Guerra Fría, el temor norteamericano pasaba por las condiciones del subdesarrollo de la región que, en su concepción, podría propiciar un giro revolucionario y un acercamiento hacia el Comunismo. De esta manera, la Alianza para el Progreso, se presentó como una serie de reformas tendientes a favorecer el desarrollo económico de los países latinoamericanos. En tanto estas medidas no generaron mayores repercusiones, la idea de la Doctrina de Seguridad Nacional (DSN) comenzó a sumar adeptos. De acuerdo con Nercesian y Rostica (2014), la DSN consideraba que, para garantizar la seguridad de la sociedad, era necesario el control militar del Estado en donde las Fuerzas Armadas se desempeñarían como garantes de los intereses nacionales. De esta manera, la idea de "seguridad interna" fue acompañada por la de "enemigo interno", en donde el Comunismo pasó a ocupar este último lugar. Así, la DSN, se convertiría en el fundamento ideológico de la intervención del Estado por parte de las Fuerzas Armadas. En este sentido, las dictaduras de Brasil en el '64, Argentina en el '66 y el autoritarismo democrático en Uruguay desde el '68, tomando a la DSN como fuente de legitimidad, marcarían la escena política nacional y latinoamericana, a través de la clausura política, la violencia y el control social. En este marco, los distintos gobiernos militares apuntaron a escindir el vínculo entre la vida política y la sociedad civil que, desde principios de los sesenta, se había incrementado notablemente. Si bien ha habido particularidades en cada caso, podría tejerse un hilo común en la acción-reacción que supuso este proceso. Así, frente al fortalecimiento del poder ejecutivo, la restricción de partidos políticos, una persecución y 
represión cada vez más aguda; la movilización, la acción y la lucha armada, emergieron desde algunos sectores de la sociedad en resistencia a las dictaduras.

De las experiencias de lucha de fines de los sesenta, cabe señalar la aparición de nuevas agrupaciones de izquierda en Argentina, Brasil y Uruguay que siguieron otras tendencias revolucionaras al caso soviético (Nercesian, 2013). En este contexto, los intelectuales, a la vez que comenzaron a interactuar con otros enfoques como el maoísmo y el foquismo; iniciaron un cuestionamiento sobre su compromiso ideológico y político. Ya no se trataba de un deber con la "verdad", sino de estar comprometido con el cambio revolucionario de la realidad social, nacional y latinoamericana. De esta manera, la noción de un intelectual con conciencia crítica de la sociedad y comprometido tanto con la vida política como con la transformación social se fue constituyendo como un rasgo característico del Cono Sur en los últimos años de la década del sesenta (Gilman, 2003). En paralelo, también se producía una amplificación de los consumos y prácticas culturales que, teniendo a la juventud como principal interlocutor, estaba anudada con un fenómeno de liberación individual, social y colectiva. En este sentido, se asistía a una época en donde no sólo se politizaba al intelectual, el estudiante o toda expresión del pensamiento y el arte, sino que también se estaba frente a un período caracterizado por la proliferación de prácticas políticas en diversos ámbitos culturales (Terán, 2013). De esta manera, los caminos de la política y la cultura hallaban una confluencia en la que diversas expresiones artísticas y literarias, se proponían como herramientas sociales e intelectuales de lucha contra el sistema capitalista, generando una creciente identificación entre la práctica simbólica y la práctica militante (Graselli, 2011).

En definitiva, los últimos años de la década del sesenta han estado atravesados por una multiplicidad de fenómenos políticos, sociales, económicos y culturales que gestaron un clima de época. Sería en este marco de la revolución como posibilidad, de emergentes teorías sobre el subdesarrollo y la dependencia, de descontento generalizado con las dictaduras y/o gobiernos autoritarios, de politización de la cultura y culturización de la política que, la Geografía del Cono Sur, en diálogo con autores como Pierre George, Yves Lacoste y Jean Tricart, llevaría adelante un cuestionamiento hacia la Geografía regional hegemónica y, en rigor, una primera expresión crítica.

\section{LA GEOGRAFÍA DE LOS SESENTA-SETENTA}

La institucionalización de la Geografía en el Cono Sur asumió al paradigma regional como forma de legitimación de la disciplina. Si bien el enfoque francés vidaliano por veces alternó con el anglosajón de Hartshorne, la región como unidad de análisis, en el marco de una ciencia proclamada como neutral y aséptica, sería predominante hasta la década de 1980. Sin embargo, hacia fines de " 60 y principios de los '70 esta forma de pensar la Geografía comenzó a evidenciar algunos cuestionamientos. En efecto, enmarcados en el clima de la época, para las diferentes comunidades geográficas del Cono Sur, se planteaba la necesidad de forjar una nueva mirada disciplinar cuyas principales dimensiones radicarían en estar al servicio de la transformación social, cuestionar la objetividad científica, convocar al compromiso político de investigadores y docentes, y denunciar las contradicciones del modo de producción capitalista (Pesce, 2017).

\section{De la teorética a la crítica}

En Brasil, el cuestionamiento a la matriz disciplinar regional de vertiente vidaliana, encontró una primera respuesta con la Geografía teorética. Ésta, de cuño neopositivista, comenzó a tener énfasis luego del golpe militar de 1964 en vinculación con los intereses desarrollistas del Estado que buscaba intervenir en el territorio para llevar adelante el proceso de modernización capitalista (Bomfim, 2007). Como contrapartida a esta Geografía utilitaria y al regionalismo francés hegemónico, desde mediados de la década del sesenta emergió una postura de 
renovación que, inserta en los movimientos de lucha y resistencia contra la dictadura y el capitalismo dependiente, buscó ser crítica tanto de la realidad social y política, como del pensamiento geográfico de la época (Vesentini, 2009). En efecto, empezó a tomar forma una Geografía que denunciaba el carácter contradictorio del desarrollo-subdesarrollo, a la vez que pretendía establecer un compromiso con la transformación de la sociedad. Esta propuesta tendría una proximidad teórica con la Geografía activa de Pierre George, junto con otros aportes de Jean Tricart e Yves Lacoste. Estos geógrafos que discutían y debatían ideas sobre la Geografía aplicada, el papel del planeamiento y la situación del subdesarrollo, formaban parte del Partido Comunista francés y serían integrantes del grupo renovador de la Geografía humana francesa de la segunda posguerra (Pedrosa, 2013). Sin embargo, a pesar de su militancia, estos autores no propondrían un vínculo epistemológico entre la Geografía y el marxismo sino más bien serían un disparador reflexivo para la denuncia y discusión (Verdi, 2016).

La idea de una Geografía activa que, desde un enfoque regional planteaba dejar de lado una labor técnica supeditada a los intereses capitalistas para pasar a identificar las tensiones y contradicciones presentes en una región, a la vez que obraba en detrimento de la que es aplicada y utilitaria al capital, colocaba al planeamiento del territorio como instrumento fundamental para logar una sociedad más justa. Para Escolar y Moraes (1989), la perspectiva de Pierre George, a pesar de realizar una ruptura política con la escuela vidaliana, asumía sus fundamentos $\mathrm{y}$, en tanto operaba como una radicalización de la reflexión posibilista, sólo realizaba una aproximación al marxismo en términos de demanda práctica y no como resultado de un esclarecimiento teórico. Por lo tanto, esta Geografía sostenía una reflexión esencialmente empirista, en la que rara vez se explicitaban los presupuestos epistemológicos y metodológicos. En rigor, si políticamente esta Geografía activa suponía una renovación respecto de la Geografía regional vidaliana, epistemológicamente, no terminaría por hacer un despegue definitivo.

Desde mediados de la década del sesenta, el Instituto Brasilero de Geografía y Estadística (IBGE), inserto en los ámbitos de la planificación territorial, supo albergar la propuesta de Geografía activa. Michel Rochefort fue un geógrafo francés que, próximo a Pierre George y partidario de su propuesta, participó activamente en los ámbitos de planificación brasileños, y en particular, en la construcción de polos de desarrollo bajo el pensamiento de François Perroux (Pedrosa, 2013). En este sentido, al interior del IBGE, se discutía el utilitarismo de la Geografía, promoviendo acciones que incluyeran la toma de acción sobre el subdesarrollo, enfatizando el compromiso político en la organización del territorio. Sin embargo, esta corriente de geógrafos de izquierda prontamente encontraría dificultades para asentarse ya que, para fines de la misma década, los cambios en la dirección del IBGE, traerían aparejado un distanciamiento de la corriente francesa y un acercamiento a la New Geography anglosajona (Bomfim, 2007).

Ahora bien, la Geografía activa de Pierre George no estuvo acotada a las instituciones vinculadas al planeamiento territorial. En efecto, en el marco de las luchas y resistencias contra la dictadura iniciada por Humberto Castelo Branco, esta vertiente práctica de la Geografía fue bien recibida en las Universidades y otros espacios de enseñanza, ya que, a través de ella, geógrafos y geógrafas, veían la posibilidad de asumir un papel y contribuir con la superación de las desigualdades sociales de Brasil (Pedrosa, 2015). De igual modo sucedía con la propuesta dialéctica de Tricart y las ideas de Lacoste respecto al imperialismo, al colonialismo y el subdesarrollo. En este sentido, si para Vesentini (2009) la lectura y debate de estos autores se daba en los ámbitos de enseñanza inicial y media; para Pedrosa (2015) la misma situación tenía lugar en la academia ${ }^{1}$.

Los estudios realizados por Tricart, como el hábitat rural o urbano, eran bienvenidos en esta renovación de la Geografía brasilera, ya que permitían superar las monografías próximas al enfoque vidaliano, por otros análisis que se centraban en las estructuras del medio, entendiéndolas como resultado de diversos cuadros históricos, económicos y sociales (Pedrosa,

\footnotetext{
${ }^{1}$ También Pedrosa (2013) destaca el uso de las ideas de Tricart en investigaciones y ámbitos de enseñanza; y Verdi (2016) remarca la referencia de George y Lacoste en la producción geográfica de la Universidad de San Pablo (USP)
} 
2013). En este marco, el trabajo de campo y la dialéctica del conflicto ${ }^{2}$ se constituían como insumos claves. Si por un lado su óptica dialéctica estaba en sintonía con una Geografía que pretendía reconocer los conflictos en los países subdesarrollados, por otro, la función del trabajo de campo, como instancia para la síntesis geográfica, podía anudarse con una Geografía regional que estaba volcada a la práctica.

Por su parte, el trabajo de Lacoste en Los Países Desarrollados (1959) y La Geografía del Subdesarollo (1965), era un análisis en clave de Geografía activa que abordaba la situación del subdesarrollo en comparación con el desarrollo, como así también a partir de la existencia de contradicciones económicas, políticas y sociales internas (Verdi, 2016). Al mismo tiempo, estas obras planteaban que la Geografía no debía limitarse a hacer un análisis aséptico, sino ser útil para la liberación de los países subdesarrollados. De esta manera, las ideas de Lacoste, que incluso podían articularse con las teorías de la dependencia y del centro-periferia que para la misma época eran enunciadas por la CEPAL, incitaban a la lucha contra el imperialismo, comprendido como parte de la lógica de sistema capitalista.

En definitiva, George, Lacoste y Tricart han estado presentes en diversos ámbitos de la Geografía de Brasil, siendo referencias teóricas de una primera renovación crítica que, a fines de la década del sesenta, se construía al reverso de la Geografía regional hegemónica y la Geografía teorética. En rigor, como afirma Pedrosa (2013: 180): "Brasil fue un paradigma y un espacio de experimentación para los geógrafos de izquierda a través de una postura que buscaba combatir el subdesarrollo activamente mediante la acción estatal, mejorando los niveles sociales incluso frente a un gobierno autoritario."

\section{La sala de Geografía del IPA y la crítica desde la docencia.}

En Uruguay, la idea de crítica comenzó a tener lugar en la Geografía a través de docentes y estudiantes que componían la Sala de Geografía del Instituto de Profesores "Artigas" (IPA). Este espacio se posicionó en el centro de los cuestionamientos llevando adelante debates y discusiones que promovían una renovación en la disciplina. Es de destacar que el espíritu renovador de este grupo estuvo enmarcado en el conjunto de movimientos sociales y políticos de fines de la década del sesenta, proponiendo que la investigación científica en general y, la geográfica, en particular, se constituyeran en un instrumento afín a la transformación revolucionaria de la sociedad (Achkar, Domínguez, Pesce, 2011).

Los estudiantes y docentes de la Sala de Geografía del IPA comenzaron a identificar algunos aspectos de la Geografía regional hegemónica considerados anacrónicos para la comprensión de la realidad nacional y latinoamericana atravesada por la dependencia y el subdesarrollo, como así también que obstruían la colaboración en el proceso de cambio social. En este sentido, se elevó una crítica contra la perspectiva naturalista, la descripción como un relato pintoresco o exótico que primaba sobre el análisis científico de la realidad, la ausencia de explicaciones de tipo histórico-social, el contenido enciclopédico y memorístico, y el vacío en la producción de conocimientos de la disciplina, entre otras cuestiones (Bruschi y Cutinella, 2016). En su lugar, este grupo proponía incluir y debatir las ideas sobre el subdesarrollo dependiente y sus contradicciones con el desarrollo, realizar estudios sobre las estructuras socioeconómicas tomando como referencia tanto la escala nacional como regional, redactar y editar materiales de estudio sustitutivos, y profundizar en las salidas de campo (Wettstein, 2014). De esta manera,

\footnotetext{
"La crítica se convierte en un instrumento metodológico superador de la Geografía tradicional, en un proceso de construcción acumulativa. De esta forma funciona como forma de poner en cuestión los fundamentos de la Geografía
}

\footnotetext{
${ }^{2}$ Tricart entiende que la dialéctica se constituye como un conflicto en el que la identidad antagonista y afirmativa de cada una de las partes se pauta en la lucha. Así, utiliza la dialéctica de la naturaleza identificando elementos del paisaje que están en conflicto, es decir el desequilibrio natural y la lucha por su reorganización estable con menor ocurrencia de fenómenos capaces de alterarlo (Pedrosa, 2013: 114)
} 
vigente, así como les va permitiendo ofrecer propuestas alternativas. A la vieja

Geografía se opone una nueva." (Bruschi y Cutinella, 2016: 59).

Ahora bien, siguiendo a Wettstein $(2014,1972)$, este proyecto de Geografía que, desde la crítica, se construía frente a la matriz regional hegemónica, también buscaría formar parte de un proyecto político más amplio, en donde geógrafos y geógrafas colaborarían con la reconstrucción de la base material de la sociedad. De esta manera, frente a las diversas dificultades que atravesaba la sociedad uruguaya bajo el gobierno autoritario de Jorge Pacheco Areco desde fines de la década del sesenta, la nueva propuesta de Geografía intentaba enmarcarse en un plan de desarrollo económico y social para la liberación nacional, poniéndose al servicio de la planificación, la investigación, y la enseñanza. En este marco, las referencias teóricas se asociaban con la Geografía activa de Pierre George, que destacaba la importancia del planeamiento como herramienta para la transformación social; y Los Países Subdesarrollados y La Geografía del Subdesarrollo de Yves Lacoste, que brindaban un abordaje de la situación del subdesarrollo. De hecho, desde estas perspectivas, la comunidad geográfica uruguaya proponía que los estudios de Geografía sean de aplicabilidad indirecta, contribuyendo con diagnósticos de la situación, denuncias de las contradicciones del sistema, e interpretaciones útiles para el cambio social. Así, esta propuesta nueva de Geografía divulgaba las virtudes de una ciencia geográfica activa, opuesta a una ciencia geográfica entendida como pasiva, contemplativa y descriptiva. Como afirmaba Wettstein (1972:9): "Un profesor de Geografía en país subdesarrollado dependiente debe tener conciencia clara de los objetivos que ha de defender (...) orientar la ciencia geográfica a la ciencia proyectada sobre un medio ambiente, para contribuir (...) a la liberación económica y social...". De esta manera, se planteaba a la Geografía como una "ciencia de la transformación de la naturaleza", que sea activa y aplicada a los propios objetivos y necesidades de los países latinoamericanos sumergidos en el subdesarrollo dependiente.

Este planteo en donde primaba la práctica por sobre la teoría, tenía un correlato en las salidas de campo que se realizaban desde la Sala de Geografía del IPA, cuya importancia radicaba en conocer el Uruguay e integrarlo bajo un abordaje nacional (Bruschi y Cutinella, 2016). De esta manera, se priorizaba un conocimiento construido desde las actividades prácticas, que encontraba apoyo en las ideas de Jean Tricart (2017), para quien el trabajo de campo, en tanto orientaba el planeamiento y la acción, era un elemento indispensable en el estudio geográfico. De esta manera, los aportes de Tricart también eran utilizados por geógrafos y geógrafas del Uruguay, en donde su óptica dialéctica aplicada en la salida de campo podía contribuir con la identificación y el análisis de las tensiones y contradicciones que atravesaban a los países subdesarrollados.

En definitiva, desde la Sala de Geografía del IPA, hacia finales de la década del sesenta, se encabezó un proceso de renovación disciplinar que tuvo la crítica a la Geografía regional hegemónica como punto de partida. Desde allí, emergió un proyecto diferente llamado Nueva Geografía (Bruschi y Cutinella, 2016) que, apoyándose en la Geografía activa de George, en las ideas sobre el subdesarrollo de Lacoste y en el método dialéctico de Tricart, construyó un campo de conocimientos que ponderaba el compromiso político con la realidad social, en donde era necesario conocer el país y entender las contradicciones albergadas en él, para encauzar el movimiento de liberación nacional. Por lo tanto, como afirma Fernando Pesce (2017:15):

\footnotetext{
"Los colectivos docentes que pretendieron reconfigurar el campo disciplinar se comprometieron en la construcción colectiva del conocimiento geográfico y establecieron las bases de un pensamiento crítico con proyección nacional y regional. Fue un momento en el que los sentidos políticos que sustentaron la construcción discursiva se basaron en una Geografía comprometida con la transformación radical de la sociedad..."
} 


\section{Las Geografías populares y la crítica en Argentina}

Desde la institucionalización de la Geografía en Argentina, uno de los grandes centros de producción del conocimiento geográfico era la Sociedad Argentina de Estudios Geográficos (GAEA). Esta organización, nucleando a docentes e investigadores, influía con un importante peso tanto en textos de enseñanza, como así también en el tono de los temas, metodologías y enfoques del quehacer geográfico. De hecho, enmarcándose en la Geografía regional de Hartshorne, la GAEA predicaba un conocimiento neutral, aséptico, despolitizado y desvinculado de otros ámbitos de conocimientos allegados a las ciencias sociales (Cicalese, 2007). También desde 1961, en el marco del proceso de modernización capitalista encabezado por el gobierno de Arturo Frondizi, se había creado una sección de Geografía aplicada que intentaba poner en contacto a esta organización con las políticas desarrollistas de la época. En rigor, la GAEA buscaba participar e influenciar en las diferentes formas de pensar y realizar Geografía.

Ahora bien, desde fines de los sesenta, esta Geografía regional hegemónica comenzó a ser cuestionada por un modelo de disciplina que buscaba orientarse hacia la profesionalización tanto en lo académico, a través de un enriquecimiento en la investigación, como en lo extraacadémico, en vías de ampliar los horizontes laborales (Iut 2005). En efecto, al tiempo que la Geografía iniciaba fecundos intercambios con la Antropología, la Economía y la Sociología, y comenzaba a ocupar un lugar clave en el planeamiento territorial; Elena Chiozza, articulando su labor en la planificación con su tarea en editoriales, vehiculizaría una propuesta diferente en la disciplina a través de Geografías Populares ${ }^{3}$, producidas por el Centro Editor de América Latina (CEAL).

Ya desde la elaboración de Mi País, Tu País, lanzada por el CEAL en 1968, Chiozza mostraba sesgos alternativos para el abordaje de la Geografía regional. Si bien esta obra estaría destinada a la formación ciudadana de preadolescentes, junto a la descripción regional se incluían pasajes literarios, cuentos y poesías, que configuraban una escritura más amena a la que ofrecía la matriz clásica (Cicalese, 2008). Sin embargo, sería a principios de los setenta que, con El País de los Argentinos, Chiozza criticaría la neutralidad planteada por la GAEA y desarrollaría una propuesta regional diferente, en donde las problemáticas sociales adquirían preeminencia en el análisis geográfico:

\footnotetext{
“[Chiozza dice]...nosotros sentíamos la necesidad de mostrar el país real. No un país libresco que se repite permanentemente igual sin que nada pasara. Parecía, por aquella época que no cabía hacer un juicio de valor, y decir esto está bien y esto está mal, uno debía mantenerse dentro de los lineamientos de una geografía aséptica." (Cicalese, 2008: 11).
}

En efecto, esta enciclopedia presentaba los conflictos sociales, políticos y culturales que se observaban en las regiones, entendidas como producciones históricas, ligadas a la forma de inserción del país en la economía nacional y mundial (Zusman y Lus Bietti, 2017). En este sentido, esta obra denunciaba las diferentes problemáticas derivadas de las desigualdades regionales en donde los análisis socioterritoriales no sólo estaban en sintonía con las teorías de la dependencia y de centro-periferia de raigambre cepaliana, sino que también evidenciaban descripciones, explicaciones y valoraciones en lo que parecía ser una clara dirección a una Geografía activa (Cicalese, 2008).

El trabajo de Pierre George era conocido gracias a las traducciones de sus libros realizadas por la Editorial de la Universidad de Buenos Aires (EUDEBA), pero también este geógrafo estuvo presente en los trabajos de consultoría de Chiozza (Cicalese 2018a). De esta manera, en vínculo con George, Chiozza destacaba el papel de la planificación para la superación de las desigualdades sociales, y desarrollaba una Geografía regional diferente a la propugnada por la

\footnotetext{
${ }^{3}$ Siguiendo a Cicalese (2018b), las Geografias Populares refieren a compendios territoriales, diccionarios y enciclopedias geográficas, como además atlas de distinta naturaleza y revistas ilustradas de geografía de corte periodístico y tirada periódica
} 
GAEA. De hecho, es interesante reparar en que Patricio Randle, geógrafo integrante de la GAEA, cuestionó El País de los Argentinos por su contenido ideológico ${ }^{4}$. Así, esta obra que mostraba intercambios epistemológicos de la Geografía con las ciencias sociales y tomaba ideas de la CEPAL, era concebida por Randle como deformadora de la imagen del país, ya que desnaturalizaba la realidad nacional y despertaba antinomias ideológicas en la sociedad (Cicalese, 2018b).

Junto a esta Geografía regional activa elaborada por Chiozza, es importante destacar la renovación académica producida en la carrera de Geografía de la, por entonces, Universidad Nacional y Popular de Buenos Aires (UNPBA). Así, en 1973, con la vuelta a la democracia luego de la dictadura militar iniciada en 1966, y la participación triunfante del peronismo tras dieciocho años de proscripción electoral, se asistía a importante proceso de politización de la sociedad argentina. Tal situación alcanzó a los ámbitos universitarios, generando un clima propicio en el campo académico para que nuevas generaciones de geógrafos y geógrafos, que también manifestaban su disconformidad con la GAEA, modificaran el plan de estudios de la carrera de Geografía. Carlos Reboratti (2019), de activa participación en tal gestión, afirmaba que se produjo una importante renovación bibliográfica que incorporaba libros y materiales tanto de Pierre George como de Yves Lacoste ${ }^{5}$. A su vez, este actor clave del período comentaba que, en este proceso renovador, no había una recepción significativa de las ideas de Jean Tricart. En este sentido, podemos concebir que, desde su disconformidad con una Geografía que consideraban neutral y aséptica, las nuevas generaciones que tomaron el control de la carrera de Geografía de la UNPBA, con aportes de George y Lacoste, planteaban un conocimiento comprometido con los sectores más humildes, en donde la planificación era vista como herramienta prioritaria de transformación social ${ }^{6}$, y la realidad nacional-latinoamericana era comprendida a través de conceptualizaciones como subdesarrollo, tercermundismo, imperialismo y dependencia (Cicalese, 2007; Iut, 2005).

En definitiva, a partir de las Geografías populares de Elena Chiozza y de la renovación académica de la carrera de Geografía en la UNPBA, es posible rastrear una propuesta que, cuestionando a la matriz regional hegemónica, denunciaba las contradicciones del sistema capitalista, atendiendo las desigualdades regionales producidas por las condiciones de desarrollo y subdesarrollo dependiente. En este sentido, destacamos la aparición de enfoques renovadores de la disciplina desde ámbitos internos y externos a la Universidad, que buscaron forjar una nueva perspectiva de la Geografía, colocando en el centro la necesidad de desarrollar conocimiento al servicio de la sociedad.

\section{ENCUENTROS Y VÍNCULOS}

La coyuntura de dictaduras y gobiernos autoritarios que se acompañó de crisis económicas, políticas y sociales, encontró un contrapunto en la Geografía del Cono Sur. En efecto, el clima de efervescencia de finales de los sesenta tuvo eco en la Geografía de Argentina, Brasil y Uruguay, a través de un proceso de renovación que buscaba cuestionar la neutralidad científica de la Geografía hegemónica, promoviendo en su lugar un conocimiento que tuviera como eje el compromiso con la realidad social, la denuncia de las contradicciones del desarrollosubdesarrollo y la lucha contra el sistema capitalista. Esta propuesta, que diálogaba con geógrafos de izquierda como George, Lacoste y Tricart, encontró una simultaneidad en el Cono Sur que se cristalizaría en el proyecto forjado por los Encuentros Latinoamericanos de la Nueva

\footnotetext{
${ }^{4}$ La crítica titulada El país de algunos argentinos, fue publicada en 1977 en el boletín de la GAEA Nº6

${ }^{5}$ La editorial EUDEBA, desde comienzos de la década del sesenta venía publicando varios libros traducidos al español de Pierre George. También, en 1962, lanza la traducción de Los Países Subdesarrollados de Lacoste. Por su parte la Editorial Ariel, desde 1966 tenía traducido el libro de George Geografía Activa, mientras que en 1971 hizo lo propio con la Geografía del Subdesarrollo de Lacoste.

${ }^{6}$ De acuerdo con Cicalese (2007), esta nueva generación tenía gran expectativa en la apuesta que hacía el gobierno popular con la planificación estatal como medida para afianzar la unidad nacional, lograr la justicia social y contribuir mediante la intervención territorial a mejorar las condiciones de vida de las regiones rezagadas
} 
Geografía. Desde allí, entrarían en relación diferentes intelectuales que establecerían vínculos de colaboración académica, llevando adelante una perspectiva geográfica de raigambre crítica y alcance latinoamericano.

\section{Los encuentros de la Nueva Geografía}

En ocasión de la trigésima-cuarta celebración de las Semanas de la Geografía, realizadas en Octubre de 1972 por la Sociedad Argentina de Estudios Geográficos (GAEA), se daría un primer contacto en geógrafos argentinos y uruguayos. Desde charlas informales, Carlos Reboratti, por ese entonces estudiante avanzado de la licenciatura en Geografía de la Universidad de Buenos Aires (UBA), y Alfredo Tróccoli junto con German Wettstein, profesores de la Sala de Geografía del Instituto de Profesores "Artigas" (IPA) y de la Universidad de la República (UdelaR), iniciaron un vínculo de coincidente disidencia con la propuesta intelectual e ideológica que la GAEA representaba (Quintero, Dufour, Iut, 2009). En efecto, existía un acuerdo en problematizar sobre la práctica científica y de docencia de la Geografía, las instituciones geográficas existentes, la formación de los geógrafos y geógrafas, y su rol en la sociedad contemporánea (Wettstein, 1973). Desde allí, comenzó a crecer el interés por construir un espacio que permitiera pensar una Geografía diferente en sus convicciones y fundamentos, como así también que tenga un alcance latinoamericano. Este deseo se concretizó en Enero de 1973 en la ciudad de Salto, en el marco de los encuentros que venía desarrollando la Asociación Nacional de Profesores de Geografía (ANPG) del Uruguay ${ }^{7}$, bajo el nombre Primer Encuentro Latinoamericano para la Nueva Geografía. Con más de cien participantes, este encuentro significaba “...un primer intercambio sistemático de ideas y experiencias entre geógrafos, profesores y estudiantes de geografía de Argentina y Uruguay que entienden que la geografía se ve necesitada de una profunda revisión, tanto en lo teórico como en lo práctico." (Wettstein, 1973: 12). En efecto, se proponía llevar adelante una Geografía de los países subdesarrollados que estuviera al servicio de las causas populares, propiciando la transformación del medio geográfico en beneficio de toda la sociedad. Para ello, se consideraba pertinente realizar un análisis crítico de los marcos teórico-conceptuales, y adecuarlos a las propias realidades del continente. También, se sostenía la necesidad de realizar un segundo encuentro, a fin de darle continuidad a esta propuesta.

Organizado por Alfredo Tróccoli, tras su exilio en Neuquén, el Segundo Encuentro Latinoamericano para la Nueva Geografía tuvo lugar en Febrero de 1974 en la Universidad Nacional del Comahue. Con la presencia de Germán Wettstein, Carlos Reboratti y Elena Chiozza, este segundo encuentro buscó consolidar las intenciones del primero. De esta manera, se planteaba reafirmar su validez como instancia para lograr una renovación de la disciplina, y se sostenía la existencia de una Nueva Geografía, destacando su papel en el proceso de liberación de los países del Tercer Mundo:

"La Nueva Geografía debe contribuir a modificar sustancialmente la organización de los espacios americanos. La historia de nuestras patrias ha demostrado que los mismos fueron organizados para servir los intereses estratégicos de las potencias mundiales hegemónicas (...) Si la Geografía es la ciencia que estudia la organización del espacio, ella debe contribuir a la definición de las soluciones referentes a aquellos desequilibrios. El geógrafo debe estar en condición de ofrecer a los centros nacionales de decisión, el estudio y la interpretación global de nuestra realidad espacial." (Segundo Encuentro Latinoamericano de la Nueva Geografía. Conclusiones, 1974: 4).

De esta manera, si por un lado se afirmaba el compromiso de la Geografía involucrada con la realidad social; por otro se valoraba su profesionalización, esto es, una vertiente práctica en la

${ }^{7}$ Desde 1967, la Asociación Nacional de Profesores de Geografía (ANPG) desarrollaba congresos de Geografía en formato bianual. El primero se realizó en Montevideo (1967), el segundo en Paysandú (1969) y el tercero en Rivera (1971). El cuarto congreso a realizarse en 1973 estaba previsto para la ciudad de Salto; y sería allí que tendría lugar el Primer Encuentro Latinoamericano para la Nueva Geografía. 
que la planificación territorial aparecía como instrumento clave para lograr el cambio social. En rigor, en este segundo encuentro se enfatizaba en la participación activa de geógrafos y geógrafas profesionales en el marco de un proceso político de liberación nacional y latinoamericana (Cicalese, 2007; Quintero, Dufour, Iut, 2009).

Pensando en clave de una Geografía activa, es interesante destacar que, para llevar adelante la conferencia inaugural del segundo encuentro, había sido invitado el propio Pierre George; situación que podría reflejar su influencia en esta propuesta de Nueva Geografía. Sin embargo, George no pudo concurrir y quien daría unas palabras iniciales sería el único representante de la delegación de Brasil: Milton Santos. Invitado por Tróccoli, Santos saludó la propuesta de estos encuentros, destacó el rol de la Geografía en la coyuntura mundial, y planteó el estudio del espacio como tema geográfico central:

\begin{abstract}
"La solución de los problemas de distribución de las riquezas producidas por la colectividad, pasará, obviamente, por el estudio del espacio. Será a través del espacio, de la manera en cómo dicho espacio sea valorizado que los diferentes países aceptaran o no distribuir o redistribuir las riquezas acumuladas. El geógrafo entonces desempeñará una misión muy destacada en la reparación de este mundo que está empezando." (Universidad Nacional de Comahue, 1974: 3).
\end{abstract}

Desde una perspectiva que avalaba y profundizaba los presupuestos básicos de la Nueva Geografía, la presencia de Milton Santos marcó sentidos y rumbos, convirtiéndose, desde allí, en una referencia ineludible para el Cono Sur (Reboratti, 1996). Si bien en la declaración final del segundo encuentro se proponía la realización de un tercero, la inestabilidad institucional argentina que derivó en la dictadura del ' 76 , terminó por diluir tal posibilidad, y con ella, esta primera perspectiva crítica de la Geografía.

Los Encuentros Latinoamericanos para la Nueva Geografía se constituyeron a principios de los setenta como un espacio que cristalizó los enfoques renovadores de la disciplina que emergían en los países del Cono Sur. Bajo una co-producción uruguayo-argentina, más tarde acompañada por Milton Santos de Brasil, este proyecto de Nueva Geografía consolidó una mirada disciplinar que, cuestionando a la Geografía regional hegemónica, bregaba por una Geografía comprometida con la realidad social, y afín a las problemáticas latinoamericanas. De esta manera, se dio una experiencia de colaboración intelectual que forjó y estrechó vínculos claves para sustentar y expandir este proyecto.

\title{
Vínculos en torno a la Nueva Geografía
}

Desde fines de la década del cincuenta, a partir del vínculo con geógrafos franceses que por ese entonces visitaban diferentes países de Latinoamérica, se abrió la posibilidad de que geógrafos y geógrafas del Uruguay pudieran acceder a becas de formación en el Centro de Geografía Aplicada de la Universidad de Estrasburgo. Quien estaría a cargo de este espacio sería Jean Tricart, y tanto Alfredo Tróccoli como Germán Wettstein tomarían sus clases e iniciarían lecturas en las obras de Pierre George e Yves Lacoste. También, desde 1956, en el marco del congreso de la Unión Geográfica Internacional (UGI) celebrado en Rio de Janeiro, Tricart estaría en contacto con la Geografía de Brasil, y en especial con Milton Santos. De hecho, años más tarde sería su tutor en el doctorado que el geógrafo brasileño realizaría en la Universidad de Estrasburgo ${ }^{8}$. De esta manera, allí en Francia se daría la ocasión para que Milton Santos se conociera con Alfredo Tróccoli, mientras que con German Westtein (1996) esto recién sucedería en Neuquén en 1974, en el Segundo Encuentro Latinoamericano para la Nueva Geografía.

En una entrevista realizada por Guillermo Cicalese (2008), Elena Chiozza afirmaba que su vínculo con Milton Santos se inició en 1963, tras conocerse en Brasil por una invitación del geógrafo brasileño Orlando Valverde. Allí, esta geógrafa argentina comentaba que, tras largas horas de conversación, concordaban con Santos tanto en la necesidad de que la Geografía

\footnotetext{
${ }^{8}$ Más tarde, tras su exilio a Toulouse, Santos también se vincularía con Pierre George e Yves Lacoste
} 
cumpla un rol protagónico en América Latina, como en la importancia de establecer un diálogo entre geógrafos y geógrafas latinoamericanas. Es interesante pensar en que tales cuestiones, también serían reivindicadas por la Nueva Geografía. Chiozza no sólo conocía a Santos, sino que, en el transcurso de la década del sesenta mantuvo un importante contacto con Pierre George, quien aportó ideas y colaboró en sus actividades de consultoría y planificación territorial $^{9}$ (Cicalese, 2018a).

Carlos Reboratti, para la misma época en que participaba en El País de los Argentinos de Chiozza, comenzaba a desarrollar la propuesta de la Nueva Geografía junto con Tróccoli y Westtein. Teniendo en cuenta que desde ambos lugares se impugnaba la Geografía predicada por la GAEA, sería posible pensar que entre Chiozza y Reboratti, y entre las Geografías Populares y la Nueva Geografía, habría algún tipo de correlato. Sin embargo, de acuerdo con el propio Reboratti (2019) si, por un lado, él no tenía un diálogo epistemológico con Chiozza, por otro, el trabajo editorial de la geógrafa argentina correría en paralelo a lo que se desarrollaba desde los encuentros. Así, a pesar de poder encontrar puntos comunes, según las palabras de este actor clave, las propuestas que emergían desde las Geografías Populares y la Nueva Geografía no habrían tenido mayor interrelación.

Como se mencionó anteriormente, el vínculo entre los geógrafos rioplatenses fue clave para el desarrollo de los encuentros de la Nueva Geografía. También, el marco de tales encuentros fue fundamental para el papel que desempeñaría Milton Santos en el Cono Sur. Gracias a la gestión de Alfredo Tróccoli, Milton Santos llegaría invitado a Comahue, y, en particular, entraría en conocimiento con German Wettstein y Carlos Reboratti. Wettstein (1996) recuerda a Milton Santos como un maestro y un amigo entrañable y, así como reconoce su relación en persona a partir del segundo encuentro, afirma que el vínculo teórico había sido trazado desde antes. En efecto, Milton Santos formaba parte de la bibliografía de un artículo en el que el geógrafo uruguayo explicitaba las ideas de la Nueva Geografía, luego del primer encuentro de Salto ${ }^{10}$. Por su parte, Reboratti $(1996,2019)$ recuerda que la influencia de Milton Santos fue primero por su presencia en Neuquén y luego llegó a través de su trabajo teórico. De hecho, es interesante reparar en que, tras el segundo encuentro, en el marco del cambio del plan de estudios de Geografía de la UNPBA en 1974, se introducirían tres obras de Santos: La Economía pobre de las ciudades de los países subdesarrollados (1971), El trabajo del geógrafo en el Tercer Mundo (1971), Geografía y Economía urbana en los países subdesarrollados $(1973)^{11}$. Sin embargo, como se ha mencionado, fueron sus palabras en el acto inaugural las que produjeron los primeros efectos:

\begin{abstract}
"Era Enero del 74. La gente estaba muy radicalizada (...) Pero saliendo de la geografía del marasmo, como podríamos llamar a esta geografía que uno había tenido en la Universidad, que viniera Milton Santos y hablara de "marxismo y geografía", nos parecía una cosa extraordinaria realmente...” (Gorojosvky, 1984:10).
\end{abstract}

Si bien no hay ponencia escrita de Milton Santos, ateniéndonos a las palabras de Reboratti, y en un repaso de la bibliografía producida por el geógrafo brasileño, es probable que sus dichos hayan estado relacionados al artículo Geografía, Marxismo y Subdesarrollo (1974) publicado en

\footnotetext{
${ }^{9}$ Jean Tricart también colaboró en actividades de consultoría y planeamiento en Argentina entre 1968 y 1971 , más precisamente en la elaboración de informes técnicos con el Instituto Nacional de Tecnología Agropecuaria (INTA), financiado por el propio ente cuando Horacio Giberti estaba al frente de la Secretaría de Agricultura y Ganadería de la Nación (Quintero, Dufour, Iut, 2009). Sin embargo, no hemos podido encontrar información que nos permita dar cuenta de una relación entre Tricart y geógrafos o geógrafas argentinas que hayan tenido participación del proyecto de la Nueva Geografía

${ }^{10}$ En el artículo titulado Una Geografía de los países dependientes. Hacia la definición de su marco teórico-práctico (1973), Wettstein utilizaba como referencia bibliográfica Le métier de géographe en pays sousdévloppé (1971), obra en la que Santos proponía la comprensión global del subdesarrollo a partir de analizar el impacto de la modernización capitalista sobre este tipo de países (Grimm, 2011). Como reflejo de esta relación, también es posible destacar por parte de Milton Santos la inclusión de un artículo de Wettstein titulado A geographic questioning from exploited countries en el $\mathrm{N}^{\circ} 9$ de la revista Antípode editada por el propio Santos y Richard Peet en 1977 (Pedrosa, 2018).

${ }^{11}$ No menos importante es que, como parte de esa nueva gestión en la UNPBA, Santos fue invitado a dar una charla: "Milton justo en ese momento estaba pasando por Buenos Aires y lo invitamos a dar clase en una especie de anfiteatro donde estaba el viejo edificio del Hospital de Clínicas, frente a Facultad de Medicina. Y Milton habló en una especie de círculo con todos sentados y él en el medio. Fue muchísima gente, estábamos muy entusiasmados.” (Reboratti, 2019).
} 
la revista Antípode. Allí, Santos realizaba una suerte de estado del arte de autores e ideas que contribuirían al debate marxista en la Geografía, destacando a George, Tricart, Lacoste y Harvey, entre otros. También, en rechazo a una ideología global, Santos bregaba por la elaboración de una teoría del subdesarrollo propia de los países del Tercer Mundo, que descansara en el método dialéctico y en la comprensión del espacio como una totalidad. De esta manera, a diferencia de otros geógrafos que vinculaban la Geografía y el marxismo, aquí, este último dejaba de aparecer como elemento partidario, para ser parte de una reflexión epistemológica. Como señala Reboratti (2019): "Milton era explícito en buscar encajar marxismo y geografía".

Ahora bien, la importancia de analizar estos vínculos radica en poder conocer con mayor profundidad de qué manera se fue construyendo este proyecto de la Nueva Geografía. En este sentido, recapitulando este último apartado, es posible distinguir dos etapas de estas relaciones. Una primera que tiene lugar desde principios de la década del sesenta, en donde comienzan a esbozarse vínculos personales y teóricos entre geógrafos y geógrafas del Cono Sur con algunos geógrafos franceses de izquierda. En Argentina, mientras Chiozza entabla relación con Pierre George, éste es leído en los espacios académicos que luego incorporan a Lacoste; en Uruguay, Tróccoli y Westtein se vinculan con Tricart y lo introducen con George y Lacoste en las lecturas de la Sala de Geografía del IPA; y en Brasil, Tricart es tutor de Santos, y junto a George y Lacoste son referencias en la producción geográfica en diversas instituciones. Estos autores, son las bases teóricas con las que se inician las críticas y propuestas renovadoras de la Geografía que convergen en el proyecto de la Nueva Geografía. Por otro lado, es posible reconocer una segunda etapa que tiene lugar a principios de los setenta con los Encuentros Latinoamericanos para la Nueva Geografía, en donde los lazos pasan a forjarse y estrecharse entre propios geógrafos y geógrafas del Cono Sur. En efecto, tras el vínculo entre Alfredo Tróccoli, German Wettstein y Carlos Reboratti se realizaron tales encuentros que incorporaron a Milton Santos como representante de Brasil. Desde su llegada a Neuquén, Santos y sus obras, también se convertirían en una referencia teórica para la Geografía del Cono Sur. De esta manera, podemos concebir que, a través de Tróccoli, Westtein, Reboratti y Santos, se configuraron vínculos importantes que favorecieron la circulación de ideas y materiales para retroalimentar este proyecto de una Nueva Geografía. Por lo tanto, en esta segunda etapa, es posible dar cuenta de una propuesta geográfica que, habiendo partido desde la crítica a la Geografía regional hegemónica, presentaba como novedad el dialogo entre sus propios integrantes para poner en práctica un conocimiento latinoamericano y comprometido con las necesidades del Cono Sur. Si los encuentros de la Nueva Geografía pueden ser señalados como acontecimientos claves en la gestación de una Geografía crítica en América Latina, entre otras razones, se debe a que detrás de ellos subyacían vínculos que sustentaron y expandieron los horizontes de este proyecto.

\section{CONSIDERACIONES FINALES}

A partir del análisis realizado, concebimos pertinente realizar unas últimas consideraciones en relaciones a dos cuestiones: por un lado que el estudio de la configuración de los vínculos entre geógrafos y geógrafas del Cono Sur nos permiten realizar rupturas con los nacionalismos metodológicos, postulando en su lugar la construcción de conocimiento a través de redes transnacionales y transfronterizas; y por otro, plantear que mientras el proyecto de Geografía de los setenta era concebida por sus intérpretes como "nueva"; a la luz del presente, creemos que es posible comprenderla como una primera expresión de la Geografía crítica.

De acuerdo con Zusman (2012), diversos análisis sobre el proceso de institucionalización de la Geografía han partido y siguen partiendo de considerar el contexto nacional estatal como el único posible y válido para estudiar estos procesos. De esta manera, la idea de que la Nación, el Estado y la Sociedad son la forma social y política natural del mundo moderno, encarna lo que Wimmer y Glick Schiller (2002) acuñan como nacionalismo metodológico, y cuyas 
implicancias para la historia social de la Geografía radican en la aceptación de que el Estado Nacional es el continente natural de la producción de discursos y conocimientos. Ahora bien, tal y como se ha explicitado en el presente trabajo, la configuración de una Nueva Geografía en el Cono Sur a principios de la década de setenta, entendida por nosotros también como crítica, sólo es posible comprenderla a partir de los encuentros y vínculos forjados entre las comunidades geográficas de Argentina Brasil y Uruguay. En rigor, fue a través de las relaciones entre Alfredo Tróccoli, German Westtein, Carlos Reboratti y Milton Santos que los Encuentros Latinoamericanos para la Nueva Geografía, fueron espacios que consolidaron esta propuesta de un proyecto disciplinar diferente. Como se ha señalado, si para fines de los sesenta primaban vínculos con referentes teóricos europeos; para principios de los setenta, la novedad radica en lazos e intercambios propiciados por propios geógrafos y geógrafas del Cono Sur. Por lo tanto, comprendemos que, a través del análisis de la Nueva Geografía, es posible dar cuenta de un proyecto disciplinar que se construyó transgrediendo los límites de los Estados Nacionales cuya base residía en vínculos académicos transfronterizos a través de los cuales circularon personas, ideas, teorías y materiales. Así, mientras que por un lado, las concepciones y obras de George, Lacoste y Tricart fueron aceptadas e incorporadas para comprender la situación y las necesidades de los países subdesarrollos; por otro, el acercamiento entre intelectuales de la Geografía del Cono Sur posibilitó la concreción de un proyecto latinoamericano para hacer de la Geografía una disciplina afín a la transformación social y a los movimientos de liberación nacional. En definitiva, concebimos que es necesario romper con el/los nacionalismo(s) metodológico(s) y tomar en consideración la construcción de espacios de producción de conocimiento transnacionales a través de los cuales se mantienen relaciones transfronterizas regulares, sistemáticas y sostenidas en el tiempo. Posicionarnos de esta perspectiva puede contribuir con la historia social de la Geografía al multiplicar las formas de entender los procesos de producción, circulación, difusión y recepción de conocimientos.

Ahora bien, en relación a la segunda cuestión consideramos relevante plantear que, en el marco de la historia social de la disciplina, la indagatoria al pasado muchas veces se entremezcla con elementos del presente. Esta cuestión, ya señalada por Livingstone (1992), sugiere siempre hacer el intento por distinguir las auto-adscripciones o auto-denominaciones de determinados fenómenos o procesos por parte de los actores involucrados, de aquello del pasado que, desde el presente, comprendemos bajo otras significaciones. Concretamente, mientras que quienes llevaron adelante esta propuesta disciplinar de la Geografía en el Cono Sur la concebían como Nueva Geografía; desde nuestro presente, a la luz del recorrido que ha trazado la historia social de la disciplina, creemos que es posible hablar de ella como una primera expresión crítica de la Geografía. Con este planteo no buscamos encasillar momentos de la historia disciplinar en ciertas categorías; sino más bien poder ampliar su comprensión.

De acuerdo al análisis realizado a lo largo de las páginas anteriores, es posible concebir que las referencias teóricas de esta Geografía de principios de los setenta no tenían un diálogo con el marxismo más que a través de la práctica. Como se ha mencionado, si la Nueva Geografía encontraba algún apoyo en las ideas de Pierre George, Yves Lacoste y Jean Tricart, estos autores invitaban a una propuesta de denuncia más que un proyecto epistemológico. En otras palabras, utilizaban el marxismo como fuente de práctica y compromiso más que como marco teórico. En rigor, la Nueva Geografía no construyó una teoría geográfica ni marxista, sino que buscaba denunciar las problemáticas socio-espaciales provenientes del subdesarrollo y en rigor, de la forma organización del mundo capitalista. A su vez, tanto los documentos del segundo encuentro de la Nueva Geografía, como así también algunos de sus actores, como Carlos Reboratti (2019), coinciden en señalar la ausencia de la palabra "crítica". De hecho, en sintonía con lo mencionado, para Quintero, Dufour y Iut (2009), antes que crítica se hablaba de 
renovación, en donde el esfuerzo estaba en superar la matriz regional hegemónica a través de la práctica más que por la teoría o el discurso.

Ahora bien, ¿Qué entendemos por crítica?, o más precisamente ¿Qué sería una Geografía crítica?. Si seguimos a Vesentini, (2009), las ideas o teorías críticas no existen en sí, sino que las mismas se definen contextualmente. En este marco, la crítica podría concebirse como una actitud reflexiva que busca comprender los fundamentos y limitaciones de teorías, ideas y pensamientos, superándolas a través de una síntesis o nueva teorización. De hecho, para este autor la crítica también puede entenderse "...como un compromiso en algún proyecto de liberación que amplíe el espacio de la democracia, que combata todas las formas de dogmatismo y de autoritarismo..." (p.116). Sosteniendo que no había un proyecto epistemológico desarrollado por la Nueva Geografía, a partir de estas ideas de Vesentini, abrimos la posibilidad a pensar en tal propuesta como un primer esbozo de la Geografía crítica.

El proyecto de Nueva Geografía partía de un cuestionamiento a la matriz regional clásica para dar cuenta de las problemáticas provenientes del subdesarrollo y de la forma de organización del mundo capitalista. En su lugar, postulaba la puesta en práctica de un conocimiento que, utilizando al planeamiento como herramienta, sirviera para llevar adelante la transformación social. En este sentido, si también asumimos que sus bases teóricas residían en la Geografía activa, podríamos concebir un planteo geográfico que, al igual que el hegemónico, se enmarcaba en la región, pero al reverso, buscaba romper con la neutralidad científica procurando contribuir con la superación de las desigualdades regionales, y con el ideal de lograr una sociedad más justa. Con esto, no queremos ni podemos sostener que desde la Nueva Geografía se elaboraba una teoría superadora; pero quizás sería posible considerar que, sin salir del encuadre regional, se reconocían las limitaciones de la Geografía hegemónica y, al menos desde las intenciones, se proponía desarrollar una perspectiva diferente. Al mismo tiempo, esta propuesta se constituía desde una disconformidad en la que se ponía como tema central el compromiso político con la realidad social. Así, actuando en contra de las dictaduras y los gobiernos autoritarios, la Nueva Geografía también buscaba contribuir con los diversos movimientos de liberación.

Desde este planteo no se pretende afirmar que el proyecto de Geografía de fines de los sesenta y principios de los setenta sea concebido como un conocimiento crítico, tal y como se entiende este movimiento a partir de la década de los ochenta y noventa, en un estrecho diálogo con la teoría social y el marxismo. La intención es abrir la posibilidad de identificar y conocer otras Geografías críticas en determinados marcos históricos. Así, en tanto hay un contexto social, político, cultural e intelectual que acompaña un cuestionamiento a la Geografía hegemónica y desde el cual se forja una Geografía alternativa con fines diferentes; pensamos que este proyecto de Nueva Geografía puede concebirse como una crítica antes de la Geografía crítica. En rigor, consideramos que a principios de los setenta tuvo lugar en el Cono Sur una primera expresión de la Geografía crítica que también podría haber servido de antesala a la Geografía crítica desarrollada en las décadas siguientes. En un intento de apertura, dejamos planteada esta idea cuya utilidad esperamos que radique en debatir sobre la historia social de la Geografía.

\section{REFERÊNCIAS}

ACHKAR, M.; DOMÍNGUEZ, A. Pesce, F. (2011) El pensamiento geográfico en Uruguay. Montevideo: LDSGAT. Facultad de Ciencias. UdelaR

ANDREU ABELA, J. (2003) Las técnicas de Análisis de Contenidos. Fundación Centro de Estudios Andaluces. Departamento de Sociología. Universidad de Granada.

BERNETE GARCÍA, F. (2013) Análisis de Contenido (Cuantitativo y Cualitativo). En: Conocer lo Social: Estrategias y Técnicas de Construcción y Análisis de Datos. Marín, L y Noboa A. Coords. Cap. 7. Madrid. 
BOMFIM, P. R. (2007). A ostentação estatística (um projeto para o território nacional: Estado e planejamento no período pós-64). São Paulo: Tese de doutorado na área de geografia humana - FFLCH/ USP. Recuperado de http://www.teses.usp.br/teses/disponiveis/8/8136/tde-11122007-100305/pt-br.php

BRUSCHI, R. Y.; CUTTINELLA, C. (2016) La Sala de Geografía del IPA y la renovación discursiva en el pensamiento geográfico uruguayo 1965-1974. Montevideo: Grupo Magro Editores.

CICALESE, G. (2007). Ortodoxia, ideología y compromiso político en la geografía argentina en la década de 1970. Barcelona: Biblo 3W, vol. XII, $\mathrm{n}^{\mathrm{o}} 767,20$ de dic. Recuperado de: http://www.ub.edu/geocrit/b3w-767.htm

CICALESE, G. (2008). La Geografía como oficio y magisterio: 'Entonces, uno se queda con la satisfacción y dice: misión cumplida, la lección fue aprendida'. Entrevista a la geógrafa Elena Margarita Chiozza. Notas, comentarios, recuadros y citas del entrevistador. Geográficos. Boletín, v.4, pp. 1-31. Recuperado de: http://nulan.mdp.edu.ar/1446/.

CICALESE, G. (2018a). Elena Chiozza. Un itinerario intelectual entre la docencia, la consultoría y las geografías populares. En Terra Brasilis (Nova Série) Revista da Rede Brasileira de História da Geografia e Geografia Histórica Nro 10 Dossiê Trajetórias de Geógraf@s 2. Recuperado de: https://journals.openedition.org/terrabrasilis/2492

CICALESE, G. (2018b). Geografías populares en la Argentina en la segunda mitad del siglo XX. Un recorrido desde La Argentina Suma de Geografía hasta el Atlas Total de la República Argentina. En Terra Brasilis (Nova Série) Revista da Rede Brasileira de História da Geografia e Geografia Histórica Nro 10 Dossiê Trajetórias de Geógraf@s 2. Recuperado de http://journals.openedition.org/terrabrasilis/3076

ESCOLAR, M.; MORAES, A.C.R.(1989). Pierre George: compromiso político, fragilidad teórica y temática regional. Anales del II Encuentro de Geógrafos Latinoamericanos. Montevideo: p.173-184.

GILMAN, C. (2003). Entre la pluma y el fusil. Debates y dilemas del escritor revolucionario en América Latina. Buenos Aires: Siglo Veintiuno editores

GOROJOVKSY, N. (1984). Nuestro departamento. Una charla con Carlos Reboratti. En Revista de los Estudiantes de Geografía| N². Facultad de Filosofía y Letras. Universidad de Buenos Aires. Pp. 4-13.

GRIMM, F.C.A (2011). Trajetória epistemológica de Milton Santos. Uma leitura a partir da centralidade da técnica, dos diálogos com a economia política e da cidadania como práxis. (Tese de Doutorado em Geografia Humana). Programa de Pós-Graduação em Geografia Humana, Universidade de São Paulo. Recuperado de: http://www.teses.usp.br/teses/disponiveis/8/8136/tde-26062012-143800/pt-br.php>

GRASELLI, F. H. (2011). Rodolfo Walsh y Francisco Urondo, el oficio de escribir. Tensiones y respuestas de una literatura peligrosa: prácticas estético- políticas y escritura testimonial. Tesis para optar por el título de Doctora en Ciencias Sociales. Buenos Aires: FCS, UBA. Recuperado de http://www.riehr.com.ar/archivos/Tesis/tesisarchivonicograsselli.pdf

IUT, V. (2005). La formación del campo profesional de geógrafos en la Argentina 1947-1975. Tesis de Licenciatura. Buenos Aires: FFYL, UBA.

LIVINGSTONE, D. (1992). The Geographical Tradition. Episodes in the History of a Contested Enterprise. Oxford: Ed. Blackwell.

NECERSIAN, I. (2013). La política en armas y las armas de la política: Brasil, Chile y Uruguay 1950-1970. $1 \mathrm{a}$ ed. CABA: CLACSO. Recuperado de http://biblioteca.clacso.edu.ar/clacso/coediciones/20130814033931/Nercesian.pdf

NERCESIAN, I.; ROSTICA, J. (2014). Todo lo que necesitas saber sobre América Latina. Buenos Aires: Paidós.

PEDROSA. B.V. (2013). Entre as ruinas do muro: a história da geografia crítica sob a ótica da ideia de estrutura. (Tese de Doutorado em Geografia Humana). Programa de Pós-Graduação em Geografia Humana, Universidade de São Paulo. Recuperado de: http://www.teses.usp.br/teses/disponiveis/8/8136/tde24092013-102013/pt-br.php

PEDROSA, B. V. (2015). A controvérsia da Geografia crítica no Brasil. Geosul, Florianópolis SC, v. 30, n. 59, p.7-27, jan/jun. Recuperado de: https://periodicos.ufsc.br/index.php/geosul/article/view/2177$5230.2015 \mathrm{v} 30 \mathrm{n} 59 \mathrm{p} 7 / 30127$ 
PEDROSA, B. V. (2018). O périplo do exílio de Milton Santos e a formação de sua rede de cooperação. História, Ciências, Saúde - Manguinhos, Rio de Janeiro, v.25, n.2, abr.-jun., p. 429-448. Recuperado de: https://dx.doi.org/10.1590/s0104-59702018000200008

PESCE, F. (2017). A tres voces. Diálogos paradigmáticos en la Geografía a fines de la década de 1960. Un tributo a la Geografía Crítica Nacional. Montevideo: UdelaR.

QUINTERO, S.; DUFOUR, E. e Iut, V. (2009) Los Encuentros de la Nueva Geografía y el surgimiento de la geografía crítica en Uruguay y Argentina durante los años '70. Ponencia. Montevideo: XII EGAL.

REBORATTI, C. (1996). El 'efecto Milton': Milton Santos y la geografía en la Argentina. En: Souza, Maria Adélia Aparecida de (org.) O mundo do cidadao. Um cidadao do mundo, pp.208-214. San Pablo: Hucitec.

REBORATTI, C. (2019) Comunicación personal. 27 de Mayo de 2019. Buenos Aires.

Santos, M. (2015) [1974] Geografia, marxismo e subdesenvolvimento. GEOUSP - Espaço e Tempo, São Paulo, v. 19, n. 1, p. 166 - 172. Recuperado de http://www.revistas.usp.br/geousp/article/view/97312

Segundo Encuentro de la Nueva Geografía (1974). Ponencias, Declaración Final, Conclusiones. Universidad Nacional del Comahue. Neuquén. Argentina.

TERÁN, O. (coord.) (2008). Ideas en el siglo. Intelectuales y cultura en el siglo XX latinoamericano. Primera edición. Buenos Aires: Siglo Veintiuno editores.

TERÁN, O. (2013) [1991] Nuestros años sesenta. La formación de la nueva izquierda intelectual argentina. Segunda edición. Buenos Aires: Siglo Veintiuno Editores

TRICART, J. 2017 [1977] O campo na dialética da geografia. Geousp -Espaço e Tempo v. 21, n. 1, p. 305-314. Recuperado de: http://www.revistas.usp.br/geousp/article/view/125762

VERDI, E. F. (2016). Produção geográfica e ruptura crítica: a geografia uspiana entre 1964 e 1985. (Dissertação de Mestrado em Geografia Humana). Programa de Pós-Graduação em Geografia Humana, Universidade de São Paulo. Recuperado de http://www.teses.usp.br/teses/disponiveis/8/8136/tde08042016-123008/pt-br.php

Universidad Nacional del Comahue (1974) Boletín Informativo. Oficina de Prensa. pp. 1-3. Neuquén, Argentina.

VESENTINI, J. W. (2009) Ensaios de geografia crítica. São Paulo: Plêiade,

WETTSTEIN, G. (1973). Una geografía de los países dependientes. Hacia la definición de su marco teórico-práctico. Buenos Aires: Ciencia Nueva, n²5, agosto, pp.7-13.

WETTSTEIN, G. (1972) [1967] La Geografía como docencia. Segunda Edición. Montevideo: Ediciones de la Banda Oriental.

WETTSTEIN, G. (2014) [1974]. "Mi” Geografía hace 40 años. Montevideo: Geoespacio, n45, abril, pp. 2-10. Publicado originalmente como Una geografía para la liberación. Montevideo: Revista de la Educación del Pueblo, n²0, mayo de 1974, pp.52-58.

WETTSTEIN, G. (1996) Una conversación con Milton Santos (que ayuda a conocerlo y a conocernos mejor). En: Souza, Maria Adélia Aparecida de (org.) O mundo do cidadao. Um cidadao do mundo, pp.475-484. San Pablo: Hucitec.

WIMMER, A., Glick Schiller, N. (2002), Methodological nationalism and beyond: national state building, migration and social sciencies. Global network 2 (4), 301-334.

ZUSMAN, P. (2012) Espacios nacionales y transnacionales en la historia disciplinar. Hacia la comprensión de la circulación de los científicos y su repercusión en el viaje de las ideas. En Cecchetto, G., Zusman, P. La Institucionalización de la Geografía en Córdoba. Contextos, instituciones, sujetos, prácticas y discursos (1878 - 1984). pp. 55-71 Córdoba: Editorial de la Universidad Nacional de Córdoba.

ZUSMAN, P.; LUS BIETTI, G. (2017) La trayectoria de la(s) Geografía(s) en Argentina. Historia de la Geografía en América Latina y el Caribe. Eds. Héctor F. Rucinque, Osvaldo Muñiz-Solari y Jorge L. Zapata Salcedo. Asociación Colombiana de Geógrafos (ACOGE). Colombia. (En prensa). 